\title{
Urinary Tract Infection in Children: An Update
}

\author{
S.M.E. Finnell ${ }^{*}$
}

\author{
Department of Pediatrics, Section for Children's Health Services Research, and Ryan White Center for Pediatric \\ Infectious Disease, Indiana University School of Medicine, Indianapolis, Indiana, USA
}

\begin{abstract}
The 2011 American Academy of Pediatrics (AAP) Urinary Tract Infection (UTI):Clinical Practice Guideline for the Diagnosis and Management of the Initial UTI in Febrile Infants and Children 2-24 Months guideline addressed "areas for research" identifying multiple areas where evidence at the time of the publication was lacking. This review discussed the evidence development in those identified areas. In this paper, I will review the latest literature on UTI in children and focus on those areas of research suggested in these guidelines. I will also summarize the related literature since September 2011.

Literature related to first UTI in children have contributed important new knowledge since the publication of the 2011 AAP guideline; 1) additional research has failed to clarify the relationship between childhood UTI and adult renal function. 2) High grade vesicoureteral reflux (VUR) has been established as a clear risk factor for scarring, but the condition is rare. Abnormal ultrasound, and fever in combination with non-E coli infection are other important predictors of scarring. 3) Antimicrobial prophylaxis appear to decrease UTI recurrences, but a large amount of antibiotics has to be administrated to prevent one UTI and the prevention works best in children with low grade, not high grade, VUR, 4) cranberry juice may prevent UTI, and 5) new, less aggressive guidelines, seem not to have negative consequences for pediatric patients measured as missed true pathology. Future guidelines would benefit from incorporating this new information.
\end{abstract}

Keywords: Chronic kidney disease, cystourethrogram, renal and bladder ultrasound, Urinary tract infection, renal scarring, voiding.

\section{INTRODUCTION}

UTI is a common cause of illness in children, with a prevalence of approximately $5 \%$ among children $2-24$ months of age with fever without obvious source [1]. As the presentation of UTI in young children are often nonspecific, and reliable urine specimens for culture require invasive methods, the diagnosis may be clinically challenging. With the goal of guiding clinicians and avoiding delays in diagnosis and treatment, multiple guidelines from numerous sources have been published over the last few years [1-4].

In 2011, the Subcommittee on Urinary Tract Infection, American Academy of Pediatrics (AAP) published the Clinical Practice Guideline for the Initial UTI in Febrile Infants and in Children of 2-24 Months [1]. The guideline is a revision of the 1999 practice parameter [5] on the same topic and presents seven action statements addressing recommendations for diagnosis, management, and follow up of UTI in young children. The publication is also accompanied by a technical report outlining the current evidence for listed action statements [6]. The most controversial change in the recommendations was to recommend a renal and bladder ultrasound (RBUS) for all children after the first UTI, but no longer routinely

*Address correspondence to this author at the Children's Health Services Research, Indiana University School of Medicine, HITS Building, Rm 1020B, 410 West 10th St., Indianapolis, IN 46202, USA; Tel: 317-2780552; Fax: 317-278-0456; E-mail: sfinnell@iu.edu recommend a voiding cystourethrogram (VCUG). This change has spurred plenty of debate in the medical community [7-9].

In addition to providing updated recommendations and the evidence for those, the 2011 AAP guideline also addressed "areas for research", identifying multiple areas where evidence at the time was lacking. What follows in this review is the evidence development that has occurred in those identified areas.

\section{CHILDHOOD UTI AND RISK OF ADULT RENAL FAILURE}

The link between childhood UTIs and the consequences for adult renal function had not been well established in 2011. Retrospective studies had addressed selected populations [6]. Ecological data from Australia [10] had demonstrated no changes in the rates of hypertension and renal failure since the widespread introduction of antimicrobial prophylaxis. A prospective cohort of children from birth until late middle age is still missing, but several recent publications have added some additional evidences in this area. In 2011, Salo et al. [11] published a study on the association between childhood UTI and chronic kidney disease (CKD). First, the authors conducted a literature search from 1966-2009 followed by a systematic review. Among the 1576 patients included, none had childhood UTI as the main cause of subsequent CKD. Secondly, they reviewed records of 366 patients treated or monitored for 
CKD at the Oulu University Hospital, Finland. Of the 58 patients without a specific noninfectious cause of CKD, one had recurrent UTI in childhood as a possible cause of CHD. The authors concluded that the etiological fraction of childhood UTI as main cause of CKD seems to be low.

In 2011, Round et al. [12] also published an article aiming to determine the true level of risk that a female child with first-time UTI will subsequently develop an end stage renal disease (ESRD) attributable to UTI. Data was collected from five renal registries around the world. Five different models were tested and the risk ranged from 1/154 $1 / 114,943$. The authors conclude that there is considerable uncertainty in the relationship between childhood UTI and the risk of ESRD.

Lastly, Toffolo et al. [13] published an additional systematic review in 2012. The authors reviewed 20 cohorts (3573 children) from 23 papers published 1980-2011 to determine the relationship between UTIs and blood pressure, renal function, growth and pregnancy-related complications. The authors concluded that there are not any clear data regarding long-term consequences following UTIs in childhood. Most data however, indicate that the outcome of renal function can be predicted, based on renal function at first and then follow up or during the first few visits. Only $0.4 \%$ of children with normal renal function initially presented with decreased renal function at follow up.

To summarise the additional data published fail to detect a strong relationship between childhood UTI and decreased renal function in adults.

\section{RISK FACTORS FOR RENAL SCARRING}

Renal scarring is known to occur both, in the presence and absence of VUR, but the degree of importance of VUR as a predictor of scarring was not well understood in 2011 .

Since then, Snodgrass et al. [14] published a crosssectional observational study of 565 patients referred for urological assessment of febrile UTI and/or VUR. Most patients $(80 \%)$ had no evidence of renal damage determined by dimercaptosuccinic acid (DMSA) scintigraphy. All grades of VUR however, increased risk for focal cortical defects versus no VUR. Grades IV and V VUR had much greater likelihood of scarring as compared to patients with grade IIII VUR.

An additional important paper on this topic was published in 2014 by Shaikh et al. [15]. The authors examined the association of predictor variables assessed at the time of a first UTI and the development of renal scarring. Studies with positive findings of a urine culture, defined by the recovery of any organisms from a suprapublic specimen, more than 10,000 colony-forming units $/ \mathrm{mL}$ from cathetercollected specimen, and more than 100,000 colony-forming units $/ \mathrm{mL}$ from a clean-voided or a bag specimen, were included. Aiming to a non biased result, studies focused only on highly selected subgroups of children with UTI were excluded, including studies of only neonates. The authors searched MEDLINE and EMBASE and identified 23 studies meeting inclusion criteria. For $9 / 23$ studies the original authors, and if necessary co-authors, responded to e-mail requests for individual level data. The authors then pooled this information for children between the age of $0-18$ years. The obtained data was used in a prediction model including age, sex, measured temperature at the time of diagnosis, duration of fever before presentation, grade of VUR, the organism isolated from culture (Escherichia coli vs other), results of RBUS (normal $v s$ abnormal), and inflammatory markers (C-reactive protein and poly morphonuclear cells, erythrocyte sedimentation rate, procalcitonin). Data on the year of enrollment as well as method of urine collection (bag vs no bag) were also gathered. The primary outcome in the article were presence of scarring, defined as any photopenia on a scan obtained at least 5 months after the initial UTI. Univariate and multivariate analysis were used in the development of the predictor model.

In the end, 1280 children were included in the analysis. $64.2 \%$ were girls and $83.7 \%$ were younger than 24 months. Renal scarring was present in 199 (15.5\%) of children. The risk factors associated with renal scarring were grade IV or V VUR, abnormal RBUS finding, grade III VUR, C-reactive protein of more than $40 \mathrm{mg} / \mathrm{L}$, temperature of at least $39^{\circ} \mathrm{C}$, organism other than E coli, polymorphonuclear cell count of more than $60 \%$ and grade I and II VUR, listed from most to least important. Age, sex and duration of fever before presentation were however not associated with renal scarring. The authors pointed out that grade IV and V VUR were by far the most important risk factors for renal scarring but they were found only in $4.1 \%$ of the children overall. Children with low grade VUR (grade I and II) had odds of scarring similar to those without VUR. The authors concluded that children with an abnormal RBUS result, or with a combination of high fever and a culture organism other than $\mathrm{E}$ coli are at a high risk of developing renal scarring.

\section{THE EFFECTIVENESS OF ANTIBIOTIC PROPHYLAXIS}

As previously mentioned, the most controversial recommendation in the 2011 AAP guideline was action statement 6a, "VCUG should not be performed routinely after the first febrile UTI; VCUG is indicated if RBUS reveals hydronephrosis, scarring, or other findings that would suggest either high-grade VUR or obstructive uropathy, as well as in other atypical or complex clinical circumstances" [1]. The 1999 version of the guideline had assumed a 100\% effectiveness from antimicrobial prophylaxis in preventing recurrent UTI, but as the evidence for the 2011 revision was collected, it became clear that this assumption was incorrect. Data from eight randomized controlled trials examining the effect of antimicrobial prophylaxis in children with UTI were collected. Metaanalysis failed to detect a significant difference in recurrence of pyelonephritis both in analysis of all children (age 0-18 years) as well as in children of 2-24 months of age [6]. Surprisingly, the point estimated suggested greater effect from antimicrobial prophylaxis in children without VUR, than in children with VUR. The rationale for routine VCUG was thus questioned, leading to the revised recommendation. In 2014 the results from the long awaited RIVUR [16] trial was published. RIVUR is a United States 2-year, multicenter, randomized, placebo-controlled trial involving 607 children with VUR who were diagnosed after a first or second febrile or symptomatic UTI. Primary outcome was 
the efficacy of trimethoprim-sulfamethoxazole in preventing recurrent UTI. Secondary outcomes were renal scarring, treatment failure (a composite of recurrences and scarring), and antimicrobial resistance. The included children were mostly commercially insured white female recruited after a first UTI, and ranged from 2-72 months of age, with a median age of 12 months. Adherence was measured by selfreport and was high. As many as $76.9 \%$ of participants reported to have taken the medication at least $75 \%$ of the time.

Recurrent UTI was developed in 39 of 302 (12.8\%) children who received prophylaxis as compared with 72 of 305 (25.4\%) children who received placebo. The relative risk was $0.55,95 \%$ CI $0.38-0.78$. The absolute risk reduction was $12.6 \%$. Eight children would have to be treated for 2 years with 5840 doses of antibiotics to prevent one case of febrile or symptomatic urinary tract infection. The hazard ratio was lower, indicating a better effect in children with low grade VUR versus those with high grade VUR. Outcome scans showed no significance between group differences in the incidence of renal scarring, $11.9 \%$ in the prophylaxis group and $10.2 \%$ in the placebo group. Significantly more children in the prophylaxis group presented with a trimethoprimsulfamethoxazole resistant $\mathrm{E}$ coli infection than those in the placebo group, $63 \%$ vs $19 \%$. In summary, the RIVUR trial provided important additional data on the effect of antimicrobial prophylaxis in preventing recurrent UTI. In this trial, the risk of recurrence was reduced by $50 \%$. However, a substantial amount of antibiotics have to be prescribed to prevent one recurrent infection, and the medicine works less well in children with high grade VUR. Scarring was not impacted.

\section{ALTERNATIVE TO ANTIBIOTIC PROPHYLAXIS}

Other treatments have been suggested as a desirable alternative in preventing recurrent UTI. At least two additional randomized controlled trials on the effect of cranberry used as such an alternative have been published since 2011. Salo et al. [11] randomized 263 Finnish children to cranberry juice for 6 months. There were no statistical difference in overall risk of recurrence between the two groups, $16 \%$ in cranberry group and $22 \%$ in placebo group, but children in the cranberry group had fewer recurrences. Furthermore, Afshar et al. [17] randomized 40 children to daily cranberry juice with and without proanthocyanidin and concluded that the children receiving cranberry juice with proanthocyanidin had a $65 \%$ reduction in UTI recurrences.

\section{OTHER AREAS IDENTIFIED AS LACKING EVIDENCE}

Beyond those areas addressed in this review, several additional areas for research were identified in the 2011 AAP guideline. Unfortunately, little additional data have been published on alternative techniques allowing less invasive sampling of urine, racial and genetic differences in risk of UTI and recurrence, or on optimal duration of antibiotic treatment. Such publications may of course be under review.

\section{IMPACT ON CHANGING IMAGING GUIDELINES}

Lastly, a growing body of evidence is being generated to assess the impact of the recently recommended more conservative approach regarding imaging after a first UTI. In 2011, Deader et al. [18] assessed the impact of the 2007 NICE guideline, which recommends no routine imaging, including no RBUS. The authors retrospectively reviewed 346 RBUS results. Scans were categorized dependent on whether it was appropriately or inappropriately to have been performed under the new NICE guidelines and by normal or abnormal RBUS result. In children 0-3 years of age only 1 of the 95 children was judged to have benefited from an initial RBUS scan which he/she would not receive under the NICE guideline. The 2011 AAP guideline does however recommend routine RBUS, but no routine of VCUG. That approach is supported by the Shaikh et al. [15] article where RBUS appeared as the most valuable test to predict which infants and young children will incur in renal scarring. Such an approach is also supported by the results in an article by Hannula et al. from 2011 [19]. The authors reviewed data on 1185 children who had undergone both RBUS and VCUG. They determined the total number of possibly missed pathological finding to $4.9 \%$ if VCUG were not performed. However, the majority of these findings had no long-term consequences and the authors concluded that children with UTI should be examined with RBUS alone and that VCUG should be performed "only after additional circumstances". In 2012 Pennesi et al. [20] further added to this body of work. Retrospective review of 406 children aged 1-36 months at their first UTI was performed to assess the revised Italian guideline. All patients underwent RBUS; those with abnormal RBUS and those with recurrence underwent both VCUG as well as DMSA scan. RBUS was pathological in $7.4 \%$ of children. Of the 48 VCUG performed, 14 showed VUR. Of the 14 children with VUR, 12 had abnormal ultrasound and 2 had recurrent UTI (which would have resulted in a VCUG even under the new guidelines). Scarring was detected in 6 children all of whom had grade IV VUR. The authors concluded that the revised guidelines for children with first UTI would result in a decrease in invasive examinations without compromising child health.

Lastly, La Scola et al. [21] evaluated the yield, economic, and radiation costs of five guideline algorithms (Melbourne Royal Children's Hospital, National Institute of Clinical Excellence-NICE), the "top down approach", AAP, and Italian Society of Pediatric Nephrology compared to a protocol where all the tests are done. Primary outcomes were the yield of abnormal tests, secondary outcomes were economic and radiation costs. 304 children were eligible. The top down approach showed the highest sensitivity for detection of VUR as well as scars $(76 \%$ and $100 \%$ respectively), but was also the most costly. NICE and AAP were most specific for VUR, and the Italian Society of Pediatric Nephrology was most specific for scars. NICE would have been the least costly approach and AAP would have resulted in the least radiation. Authors concluded that an aggressive protocol has a high sensitivity for detecting VUR and scarring, but carries financial and radiation costs without proven benefit. 


\section{CONCLUSION}

Literature related to first UTI in children have contributed important new knowledge since the publication of the 2011 AAP guideline; 1) additional research has failed to clarify the relationship between childhood UTI and adult renal function. 2) High grade VUR has been established as a clear risk factor for scarring, but the condition is rare and abnormal ultrasound, or fever in combination with non-E coli infection are other important predictors of scarring. 3) Antimicrobial prophylaxis appear to decrease UTI recurrences, but large amounts of antibiotics have to be administrated to prevent one UTI and the prevention works best in children with low grade VUR. 4) Cranberry juice may prevent UTI, and 5) new, less aggressive guidelines, seem not to have negative consequences for pediatric patients measured as missed true pathology. Future guidelines would benefit from incorporating this new information.

\section{ABBREVIATIONS}

$$
\begin{aligned}
& \text { AAP }=\text { American Academy of Pediatrics } \\
& \text { CHD }=\text { Chronic kidney disease } \\
& \text { DMSA }=\text { Dimercaptosuccinic acid } \\
& \text { ESRD }=\text { End stage renal disease } \\
& \text { RBUS }=\text { Renal and bladder ultrasound } \\
& \text { UTI }=\text { Urinary tract infection } \\
& \text { VUR }=\text { Vesicoureteral reflux } \\
& \text { VCUG }=\text { Voiding cystourethrogram }
\end{aligned}
$$

\section{CONFLICT OF INTEREST}

The author confirms that this article content has no conflict of interest.

\section{ACKNOWLEDGEMENTS}

Declared none.

\section{REFERENCES}

[1] Roberts KB, Downs SM, Finnell SME. American Academy of Pediatrics. Urinary tract infection: clinical practice guideline for the diagnosis and management of the initial UTI in febrile infants and children 2 to 24 months. Pediatrics 2011; 128: 595-610.

[2] NICE guidelines [CG54] Published date: August 2007 Urinary tract infection in children: diagnosis, treatment and long term management. Published, August, 2007. http://guidance.nice.org.uk/CG54 2015).
[3] Ammenti A, Cataldi L, Chimenz R, et al. Febrile urinary tract infections in young children: recommendations for the diagnosis, treatment and follow-up. Acta Paediatr 2012; 101: 451- 7 .

[4] Peters CA, Skoog SJ, Arant BS, Jr., et al. Summary of the AUA guideline on management of primary vesicoureteral reflux in children. J Urol 2010; 184: 1134-44.

[5] Practice parameter: the diagnosis, treatment, and evaluation of the initial urinary tract infection in febrile infants and young children. American academy of pediatrics. committee on quality improvement. subcommittee on urinary tract infection. Pediatrics 1999; 103: 843-52.

[6] Finnell SM, Carroll AE, Downs SM. Technical report-Diagnosis and management of an initial UTI in febrile infants and young children. Pediatrics 2011; 128: e749-70.

[7] Wan J, Skoog SJ, Hulbert WC, et al. Section on Urology response to new Guidelines for the diagnosis and management of UTI. Pediatrics 2012; 129: e1051-3.

[8] Roberts KB, Finnell SM, Downs SM. Response to the AAP Section on Urology concerns about the AAP Urinary Tract Infection Guideline. Pediatrics 2012; 129: e1054-6.

[9] Tullus K. Pediatrics: AAP recommends reduced imaging after first febrile UTI. Nat Rev Urol 2012; 9: 11-2.

[10] Craig JC, Irwig LM, Knight JF, et al. Does treatment of vesicoureteric reflux in childhood prevent end-stage renal disease attributable to reflux nephropathy? Pediatrics 2000; 105: 1236- 41.

[11] Salo J, Ikaheimo R, Tapiainen T, et al. Childhood urinary tract infections as a cause of chronic kidney disease. Pediatrics 2011; 128: 840-7.

[12] Round J, Fitzgerald AC, Hulme C, et al. Urinary tract infections in children and the risk of ESRF. Acta Paediatr 2012; 101: 278-82.

[13] Toffolo A, Ammenti A, Montini G. Long-term clinical consequences of urinary tract infections during childhood: a review. Acta Paediatr 2012; 101: 1018-31.

[14] Snodgrass WT, Shah A, Yang M, et al. Prevalence and risk factors for renal scars in children with febrile UTI and/or VUR: a crosssectional observational study of 565 consecutive patients. J Pediatr Urol 2013; 9: 856-63.

[15] Shaikh N, Craig JC, Rovers MM, et al. Identification of children and adolescents at risk for renal scarring after a first urinary tract infection: a meta-analysis with individual patient data. JAMA Pediatr 2014; 168: 893-900.

[16] Hoberman A, Chesney RW; RIVUR Trial Investigators. Antimicrobial prophylaxis for children with vesicoureteral reflux. N Engl J Med 2014; 371: 1072-3.

[17] Afshar K, Stothers L, Scott H, et al. Cranberry juice for the prevention of pediatric urinary tract infection: a randomized controlled trial. J Urol 2012; 188:1584-7.

[18] Deader R, Tiboni SG, Malone PSJ et al. Will the implementation of the 2007 National Institute for Health and Clinical Excellence (NICE) guidelines on childhood urinary tract infection (UTI) in the UK miss significant urinary tract pathology? BJUI 110: 454-8

[19] Hannula A, Perhomaa M, Venhola M, et al. Long-term follow-up of patients after childhood urinary tract infection. Arch Pediatr Adolesc Med 2012; 166: 1117-22.

[20] Pennesi M, Travan L, Peratoner L, et al. Is antibiotic prophylaxis in children with vesicoureteral reflux effective in preventing pyelonephritis and renal scars? A randomized, controlled trial. Pediatrics 2008; 121: e1489-94.

[21] La Scola C, De Mutiis C, Hewitt IK, et al. Different guidelines for imaging after first UTI in febrile infants: yield, cost, and radiation. Pediatrics 2013; 131: e665-71. 